Review

\title{
The roles of $\mathrm{Wnt} / \beta$-catenin signaling pathway related IncRNAs in cancer
}

\author{
Xiao-Yi Hu ${ }^{1,2,4^{*}}$, Ping-Fu Hou ${ }^{1,2^{*}}$, Teng-Teng Li ${ }^{*}$, Hao-Yu Quan ${ }^{1,2}$, Min-Le Li ${ }^{1,2}$, Tian Lin ${ }^{1,2}$, Jin-Jin Liu ${ }^{1,2}$, Jin \\ Bai $^{1,2}$, Jun-Nian Zheng ${ }^{1,2,3}$ \\ 1. Cancer Institute, Xuzhou Medical University, Xuzhou 221002, Jiangsu Province, China; \\ 2. Jiangsu Center for the Collaboration and Innovation of Cancer Biotherapy, Xuzhou Medical University, Xuzhou 221002, Jiangsu Province, China; \\ 3. Center of Clinical Oncology, Affiliated Hospital of Xuzhou Medical University, Xuzhou 221002, Jiangsu Province, China; \\ 4. Division of Hepatobiliary and Pancreatic Surgery, Department of Surgery, First Affiliated Hospital, School of Medicine, Zhejiang University, Hangzhou \\ 310003, China; \\ 5. Department of Gastrointestinal Surgery, the Affiliated Hospital of Xuzhou Medical University, Xuzhou 221002, Jiangsu Province, China. \\ * These authors contributed equally to this work. \\ $\square$ Corresponding authors: Jin Bai, Cancer Institute, Xuzhou Medical University, 84 West Huaihai Road, Xuzhou, 221002, Jiangsu Province, China. E-mail: \\ bj@xzhmu.edu.cn; Jun-Nian Zheng, Cancer Institute, Xuzhou Medical University, 84 West Huaihai Road, Xuzhou, 221002, Jiangsu Province, China. E-mail: \\ jnzheng@xzhmu.edu.cn.
}

(c) Ivyspring International Publisher. This is an open access article distributed under the terms of the Creative Commons Attribution (CC BY-NC) license (https://creativecommons.org/licenses/by-nc/4.0/). See http://ivyspring.com/terms for full terms and conditions.

Received: 2018.06.19; Accepted: 2018.10.07; Published: 2018.11.02

\begin{abstract}
Long noncoding RNAs (IncRNAs), with length of more than 200 nucleotides, are not translated into proteins but involved in multiple diverse diseases, especially tumorigenesis. The dysregulation of IncRNAs greatly contributes to the progression of various tumors through specific signaling pathways, including $\mathrm{Wnt} / \beta$-catenin signaling pathway, which is associated with malignant features of tumors. The interactions between IncRNAs, which have specific expression characteristics in diverse cancer tissues, and $\mathrm{Wnt} / \beta$-catenin signaling pathway, exhibit potential as novel biomarkers and therapeutic targets. In this review, we aim to present research findings on the roles of Wnt pathway-related IncRNAs and their effects on $\mathrm{Wnt} / \beta$-catenin signaling to regulate tumorigenesis in different cancer types. Results may be used as basis to develop or improve strategies for treatment of different carcinomas.
\end{abstract}

Key words: cancer, long non-coding RNA, Wnt/ $\beta$-catenin signaling pathway

\section{Role of lncRNAs in cancers}

Long noncoding RNAs (lncRNAs), a class of nonprotein coding transcripts, have a length of more than 200 nucleotides[1] and participate in chromatin remodeling, transcriptional control, posttranscriptional processing, and protein metabolism[2-4]. Scholars have focused on lncRNAs since lncRNA H19 was reported in 1990[5]. Many lncRNAs participate in the pathogenesis of different diseases[6]; such lncRNAs include H19[7] in abdominal aortic aneurysm (AAA), ROR in myocardial I/R injury[8], and DANCR in osteoarthritis[9]. With the development of bioinformatics and functional genomics, studies have found that lncRNAs exhibit significant functions in tumorigenic processes, such as cell proliferation, apoptosis, differentiation, and invasion.

Once considered as a crucial component of tumorigenesis, lncRNAs have been discovered to be associated with different cancers. For instance, MALAT-1 promotes the migration, invasion, and epithelial-mesenchymal transition (EMT) of gastric cancer (GC) cells[10]. High expression levels of HOTAIR[11] and CCAL[12] are correlated with poor prognosis in patients with CRC. Overexpression of HOTAIR is highly linked with the progression and metastasis of early-stage, surgically resected breast cancer[13]. The dysregulation of lncRNAs is associated with tumorigenesis, and their aberrant expression has important pathogenic consequences through different mechanisms. Guide lncRNAs bind 
to the regulatory or enzymatically active protein complexes and guide them to specific target gene promoters or genomic loci[14]. Scaffold lncRNAs provide central platforms for various protein complexes to tether to so they can be guided to specific location[14]. Decoy lncRNAs activate or silence genes by interacting with transcription factors or repressors to bind to the target gene promoters[15]. Moreover, lncRNAs considerably affect specific signaling pathways because they regulate cancer cell survival and death; the expression of lncRNAs indicates an active signaling event regardless of their roles in the signaling process[15]. For example, Sun et al. found that the downregulation of lncRNA RP11-708H21.4 promoted colorectal cancer (CRC) tumorigenesis through the AKT/mTOR pathway[16]. Meanwhile, metaLnc9 could activate the AKT/mTOR pathway to facilitate the metastasis of nonsmall cell lung cancer (NSCLC)[17].

Increasing lines of evidence have confirmed that lncRNAs are involved in various cellular processes and appear to be tissue-specific drivers of cancer phenotypes. Hence, lncRNAs could be used as predictors of patient prognosis and as treatment targets[18].

\section{Wnt Signaling Pathway}

The Wnt signaling pathway is an important extracellular pathway that was discovered in 1982 along with proto-oncogene Int1 (also known as Wnt1)[19]. This complex and ancient pathway is involved in a multitude of developmental processes, such as cell growth, differentiation, individual development, migration, genetic stability, apoptosis, self-renewal of stem cells, and maintenance of adult tissue homeostasis[20, 21].

The Wnt signaling pathway comprises two different intracellular signaling pathways: the $\beta$-catenin-dependent pathway (known as the canonical Wnt pathway) and the $\beta$-catenin-independent pathway (known as the noncanonical Wnt pathway). Compared with other signaling pathways, the canonical Wnt/ $\beta$-catenin pathway is highly evolutionary conserved and mainly involves $\beta$-catenin. In the absence of Wnt ligands, cytoplasmic $\beta$-catenin is recruited into a destruction complex containing glycogen synthase kinase $3 \beta$, adenomatous polyposis coli (APC), casein kinase (CK) $1 \alpha$, and axin and is then phosphorylated by CK1 $\alpha$ and GSK3 $\beta$. The phosphorylated $\beta$-catenin is directly ubiquitinated by $\beta$-transducin repeat-containing protein E3 ligase and degraded by the $26 \mathrm{~S}$ proteasome pathway[22] to maintain a low level of $\beta$-catenin. When the Wnt ligand binds to the frizzled receptors and coreceptor lipoprotein-related protein (LRP), the former activates dishevelled proteins to phosphorylate low-density lipoprotein receptor-related proteins 5/6 (LRP5/6), which in turn disassembles the destruction complex so the phosphorylated LRP5/ 6 provides a binding site for GSK3 $\beta$ and axin. The stabilized $\beta$-catenin accumulates in the cytoplasm and translocates to the nucleus, where it binds to the T-cell factor/lymphocyte enhancer factor (TCF/LEF) of the transcriptional complex to activate the transcription of downstream genes, such as c-Myc, cyclin D1, matrix metalloproteinase 1 (MMP-1), and MMP-7[23].

The noncanonical Wnt pathway is further divided into the Wnt/Ca2+ pathway and the Wnt/planar cell polarity (PCP) pathway. As a core mediator, the Wnt ligand is needed in both pathways to bind to frizzled receptors and LRP and subsequently activate the DSH protein[24]. Hence, Wnt proteins depend on a particular combination of Wnt receptors and coreceptors. The interaction of different Wnt ligands, which are all Cys-rich encoded by 19 Wnt genes, with different Fzd receptors would decide the downstream signaling pathway.

WNT signaling can promote or inhibit tumor initiation, growth, and metastasis and drug resistance in a cancer stage-specific or a cancer type-specific manner[25] by regulating the expression of specific target genes, such as c-myc, E-cadherin, and cyclin D1. The high expression of constitutively active $\beta$-catenin promotes the growth of myeloma cells in vitro[26]. A previous work reported a high level of nuclear $\beta$-catenin in subpopulations of leukemia stem cells that can form tumors with short latency in mice[27]. Moreover, overexpressing WNT1 induces the invasiveness of MCF7 breast cancer cells, and this effect can be attenuated by blocking $\beta$-catenin-dependent transcription[28]. Hence, identifying genetic factors and biomarkers is important for predicting responses to treatment with WNT pathway modulators, for diagnosis and determining the prognosis of the cancer, and for developing novel therapeutics.

\section{Wnt pathway-related lncRNAs and coloretal cancer (CRC)}

LncRNAs with differential expression profiles in clinical CRC tissues have been suggested to contribute to the activation or inactivation of the Wnt pathway to regulate tumorigenesis. To explore the different roles of lncRNAs in the progression of CRC via the Wnt pathway, we enumerate all of the reported Wnt pathway-related lncRNAs (Table 1). 
Table 1. LncRNAs linked with CRC via WNT signaling

\begin{tabular}{llll}
\hline LncRNA & Expression pattern & Interaction with WNT signaling & Cancer phenotype \\
\hline BCAR4 & $\mathrm{Up}$ & Activate & Promote cell proliferation, migration and inhibit apoptosis \\
CCAL & $\mathrm{Up}$ & Activate & Promote proliferation, migration, invasion and inhibit cell apoptosis \\
CASC11 & $\mathrm{Up}$ & Activate & [12] \\
DLEU7-AS1 & $\mathrm{Up}$ & Activate & Promote growth and metastasis \\
HNF1A-AS1 & $\mathrm{Up}$ & Activate & Promote proliferation, invasion, migration \\
HOTAIR & $\mathrm{Up}$ & Activate & Promote proliferation, colony formation, migration, invasion \\
H19 & $\mathrm{Up}$ & Activate & Promote proliferation and drug resistance \\
MIR100HG & $\mathrm{Up}$ & Activate & Promote MTX resistance \\
SNHG1 & $\mathrm{Up}$ & Activate & Promote cetuximab resistance \\
TCF7 & $\mathrm{Up}$ & Activate & Promote tumor cell proliferation and tumorigenesis \\
XIST & $\mathrm{Up}$ & Activate & Promote tumor cell proliferation, migration, and invasion \\
CTD903 & $\mathrm{Up}$ & Repress & Promote tumor proliferation and Invasion \\
BC032913 & Down & Repress & Inhibit tumor invasion, migration proliferation, EMT, and metastasis \\
Linc00675 & Down & Repress & [49] \\
LincRNA-p21 & Down & Repress & [31] \\
\hline
\end{tabular}

\section{Tumor promoting role of $\mathrm{Wnt}$ pathway-modulating IncRNAs in CRC}

With the development of scientific technology, increasing lines of evidence show that lncRNA-miRNA interactions mediate the functions of lncRNAs in CRC. Many CRC-related lncRNAs bind to miRNAs instead of the target mRNAs to regulate the expression of target genes[29]; some of which activate the Wnt signaling pathway and promote tumorigenesis. For example, Zhigang Xiao et al. reported[30] that IncRNA HOTAIR enhances drug resistance and promotes the proliferation of CRC cells through the miR-203a-3p-mediated $W n t / \beta$-catenin signaling pathway. LncRNA MIR100HG confers cetuximab resistance in $\mathrm{CRC}[31]$ because its two embedded miRNAs (miR-100 and miR-125b) downregulate the five negative regulators (DKK1[32], DKK[33], ZNRF3[34], RNF43[35], and APC2) of canonical Wnt $/ \beta$-catenin signaling[36, 37]. Furthermore, XIST is highly conserved to bind to miR-34a[38]. Given that miR-34a is closely linked with the 3'-UTR of WNT1, the production of WNT1 mRNA and its corresponding protein cannot be decreased in CRC due to the strong binding between XIST and miR-34a. In the presence of the WNT1 ligand, $\beta$-catenin can translocate to the nucleus to express growth- and invasion-associated genes.

Several lncRNAs, such as CASC11[39], CCAL[12], and TCF7[40], could be involved in promoting the proliferation and invasion of CRC through diverse mechanisms via the Wnt/ $\beta$-catenin pathway. CASC11[39] enhances the stability of hnRNP-K to increase its expression levels as a cytoplasmic lncRNA. By interacting with AXIN and GSK3 and forming a complex with $\beta$-catenin, hnRNP-K protects $\beta$-catenin from degradation and promotes its nuclear accumulation to activate the downstream of Wnt signaling. The ability of CCAL to enhance proliferation, cell cycle progression, invasion, and migration could be partly attributed to the fact that it inhibits AP-2a and subsequently activates the Wnt/ $\beta$-catenin signaling pathway[12]. In addition, TCF7 expression is regulated by lncTCF7 to activate WNT signaling and thus promote tumor metastasis and invasion in CRC[40].

\section{Tumor suppressor role of Wnt pathway-modulating IncRNAs in CRC}

In contrast to many oncogenic lncRNAs, lncRNAs can alter the expression and activity of essential $W n t / \beta$-catenin modulators to deregulate $W n t / \beta$-catenin signaling in CRC. For example, lncRNA BC032913 upregulates the expression of TIMP3 and inhibits the nuclear translocation of $\beta$-catenin, thereby inactivating the $\mathrm{Wnt} / \beta$-catenin pathway to suppress CRC metastasis[41]. LincRNA-p21 can directly deregulate $\beta$-catenin at the posttranscriptional level to suppress the Wnt/ $\beta$-catenin signaling pathway and enhance the radiosensitivity of CRC radiotherapy[42]. Although the oncogenic interactions between lncRNAs and miRNAs have been extensively studied, some lncRNA-miRNA interactions remain unclear. The subsequent regulation of cellular signaling pathways in CRC can be treated as potential target to suppress tumor. The negative regulatory function of miR-942 by linc00675 contributes to the inhibition of Wnt/ $\beta$-catenin signaling and the suppression of the development and progression of CRC[43] .

\section{Wnt pathway-related lncRNAs and liver cancer}

Wnt/ $\beta$-catenin signaling, which is the key regulator for the progression of liver tumors, plays a critical role in the development and stemness of cancer cells[50-53]. Diverse oncogenic lncRNAs play important roles in regulating $\beta$-catenin by several 
mechanisms for the core component of the Wnt $/ \beta$-catenin signaling pathway (Figure 1 ). The APC degradation complex and the $\beta$-catenin-TCF activating complex are the main targets to regulate $\beta$-catenin stability and activation by lncRNAs[53]. For example, lncAPC recruits EZH2 to the APC promoter and inhibits APC transcription, thereby promoting the activation of the Wnt/ $\beta$-catenin pathway and the self-renewal process of liver tumor initiating cells[54]. LncLALA1 inhibits the formation of APC degradation complex because it recruits CTCF to the axin1 promoter to block Axin1 transcription and activate Wnt/ $\beta$-catenin signaling pathway[55]. Recent research reported that lncTCF7 can activate TCF7 expression in cis by recruiting the SWI/SNF complex[56]. This evolutionally conserved multi-subunit complex consists of 12-15 subunits with several core components, such as SMARCB1/SNF5, BAF170, and BAF155[57, 58]. After the activation by the SWI/SNF complex, the overexpression of TCF7 combines with the lymphocyte enhancer factor of the transcriptional complex to activate the transcription of downstream genes in Wnt signaling, resulting in the self-renewal of liver cancer stem cells. Moreover, overexpressed linc00210 in liver cancer tissues can bind to CTNNBIP1, thereby impairing CTNNBIP1- $\beta$-catenin interaction to promote $\beta$-catenin-TCF/LEF interaction and activate Wnt $/ \beta$-catenin signaling and liver tumor initiating cell self-renewal[59]. Alterations in $\beta$-catenin activity directly influence the development of liver tumorigenesis. LncRNA $\beta$-Catm, which is associated with $\beta$-catenin, enhances the expression of zeste homolog 2 (EZH2) to promote the methylation of $\beta$-catenin and inhibit $\beta$-catenin ubiquitination[60]. The

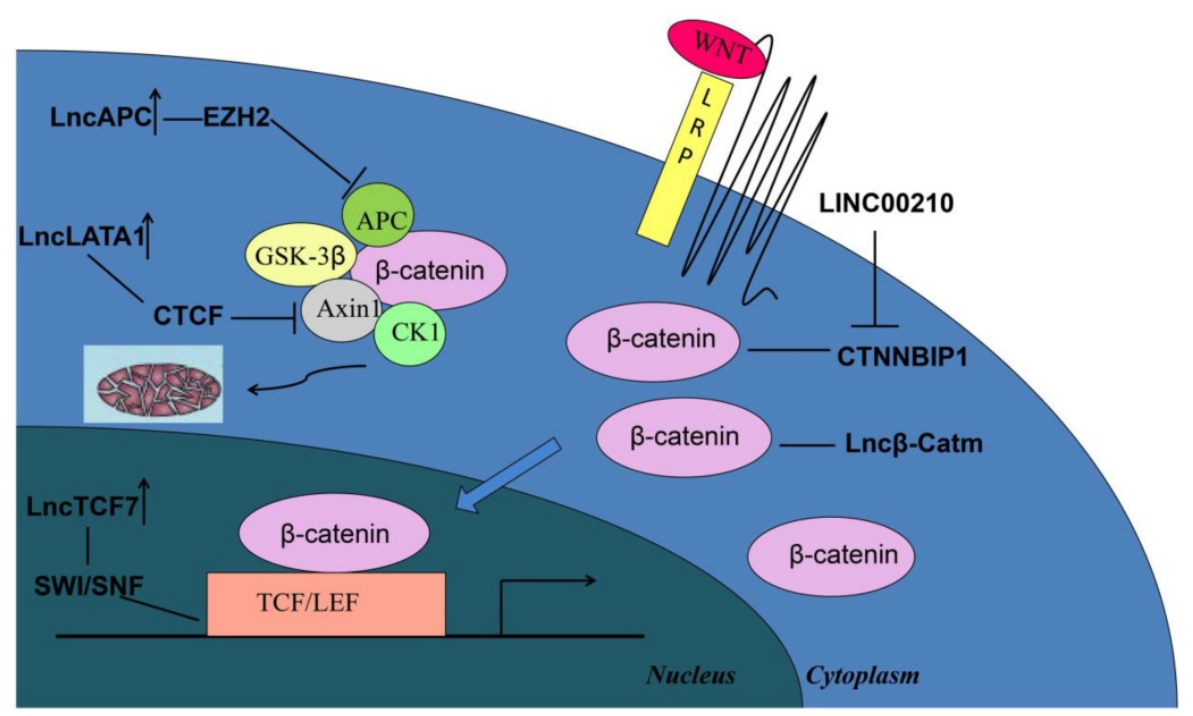

Figure 1. Schematic of the activation of $\mathrm{Wnt} / \beta$-catenin signaling by different oncogenic IncRNAs in liver cancer. Degradation and activating complexes are the two main targets for regulating $\beta$-catenin, whereas some IncRNAs may directly regulate $\beta$-catenin to influence tumorigenesis. accumulation of methylated $\beta$-catenin allows the activation of Wnt- $\beta$-catenin signaling and maintaining the stemness of liver CSCs.

\section{Wnt pathway-related lncRNAs and glioma}

Gliomas are the most common and aggressive primary brain tumors and account for approximately $80 \%$ of all cases of malignant brain tumors[61, 62]. In the past few years, the interactions of lncRNA and Wnt/ $\beta$-catenin signaling in glioma have gained increasing research attention. Several lncRNAs, such as AB073614[63], CCAT2[64], CCND2-AS2[65], and DANCER[66], have been identified to be involved in promoting tumor proliferation, migration, and invasion in glioma via the $\mathrm{Wnt} / \beta$-catenin signal pathway. The AB073614 expression increases in glioma and indicates poor prognosis; scholars have reported that the proliferation and migration of glioma cells can be increased by targeting SOX7 to activate the $\mathrm{Wnt} / \beta$-catenin signaling pathway[63]. LncRNA CCAT2 is upregulated in glioma tissues, and CCAT2 knockdown inhibits cell proliferation and tumorigenesis potential by suppressing the expression of the downstream genes of the Wnt/ $\beta$-catenin signaling pathway[64]. LncRNAs, such as CCND2-AS1[65] and DANCR[66], are correlated with poor prognosis in patients with glioma; after Wnt/ $\beta$-catenin signals are activated, tumor proliferation and invasion are promoted. These finding indicate the involvement of lncRNAs and Wnt/ $\beta$-catenin signaling pathway in glioma. However, the mechanisms on how lncRNAs activate Wnt/ $\beta$-catenin signals to promote glioma must be further explored.

Altering the expression
and activity of core
Wnt/ $\beta$-catenin modulators has
been proven as an essential
method for lncRNAs in
modulating Wnt/ $\beta$-catenin
signaling[67]. LncNEAT1As, an
essential architectural
component of paraspeckle
nuclear bodies, alters the
epigenetic landscape of target
gene promoters to promote
transcription; as such,
LncNEAT1As possesses
oncogene characteristics in
various cancers, such as breast
cancer[68], CRC[69], and
prostate cancer[70]. Studies
have demonstrated that NEAT1
functions as an oncogene in


glioblastoma, and the WNT/ $\beta$-Catenin pathway involves lncNEAT1 to promote the malignant progression of glioblastoma[71]. Furthermore, knockdown of NEAT1 reduces the trimethylation modification of H3K27 in the Axin2, ICAT, and GSK3B promoter region via EZH2. Given that Axin2, ICAT, and GS3KB are WNT-negative signaling regulatory factors[72-74], IncNEAT1 decreases the expression of the axin2/ICAT/GS3KB complex to activate Wnt/ $\beta$-catenin signaling. In conclusion, as a scaffold, lncRNA NEAT1 could bind to EZH2 and mediate the trimethylation of $\mathrm{H} 3 \mathrm{~K} 27$ in the presence of the promoters of target-specific genes (Axin2, ICAT, and GSK3B) to promote $\beta$-catenin nuclear transport, which might be a novel therapeutic target in glioblastoma.

\section{Wnt pathway-related lncRNAs and GC}

GC is the fourth most frequently diagnosed cancer[75] and the second leading cause of cancer-related mortality worldwide[76]. Although lncRNAs play an active role in cancer, their interactions with the $\mathrm{WNT} / \beta$-catenin pathway are an emerging research field in GC. LncRNA HOTAIR is negatively correlated with miR-34a level; that is, overexpression of HOTAIR decreases the miR-34a level and leads to the activation of the $\mathrm{WNT} / \beta$-catenin pathway; this phenomenon coincides with increased cellular proliferation[77]. Similarly, lncRNA FEZF1-AS1 and lncRNA LINC01606, whose expression levels are upregulated in GC cells, activate Wnt/ $\beta$-catenin signaling to promote tumor cell migration and invasion[78, 79]. LncRNA LINC01133 and ENST00000434223 are downregulated in GC tissues and cell lines, and its low expression is positively correlated with EMT and metastasis by inactivating the $W n t / \beta$-catenin pathway[80, 81]. Moreover, lncRNA zinc finger antisense 1 (ZFAS1) has different expression levels in various cancers and exerts anti-tumor or pro-tumor effects on different cancers[82-84] since it was identified in 2011. GC tissue and cell lines highly express ZFAS1, which plays a cancer-promoting role in GC[85, 86]. The underlying mechanism of ZFAS1 in promoting GC may be associated with the Wnt signaling pathway[87]. ZFAS1 recruits numerous transcriptional factors to execute its intracellular biological functions. Enhancer of zeste homolog 2 (EZH2) and LSD1 are two crucial substances that can bind to Kruppel-like factor 2 (KLF2) and NKD2 promoter regions to inhibit their transcription[86]. As a mammalian ortholog of Drosophila naked cuticle, NKD2 has frequent loss of heterozygosity in CRC and GC[88, 89] and negatively regulates canonical Wnt signaling activity by inhibiting dishevelled proteins[90]. Dishevelled proteins disassemble the complex that can destruct $\beta$-catenin by phosphorylating LRP5/6; as such, NKD2 can decrease the level of $\beta$-catenin in the cytoplasm to inhibit the Wnt signaling pathway. Hence, canonical Wnt signaling is highly activated to promote the malignancies of cancer cells by inhibiting NKD2 through overexpression of ZFAS1 in GC. Furthermore, silencing of ZFAS1 induces the inhibition of cell growth, proliferation, cell cycle, progression, migration, invasion, and EMT and enhances the sensitivity to cis-platinum or paclitaxel by suppressing canonical Wnt signaling[87].

\section{Wnt pathway-related lncRNAs and NSCLC}

Lung cancer is one of the most common causes of cancer-associated mortality worldwide[91]. NSCLC accounts for $80 \%-85 \%$ [92] of all cases of lung cancers, leading to big economic losses; the current 5-year survival rate of patients with NSCLC is <15\%[93]. Therefore, clinically useful biomarkers and therapeutic targets should be identified for diagnosis or treatment of NSCLC at an early stage. The upregulation of LINC00968 can promote the growth, migration, and invasion of NSCLC cells upon the activation of the Wnt/ $\beta$-catenin signaling pathway[94]. Multiple lncRNAs have been identified to contribute to the development and progression of NSCLC via the Wnt/ $\beta$-catenin signaling pathway. Some examples of lncRNAs are NEAT1[95] and FOXD2-AS1[96], which are also related to Wnt/ $\beta$-catenin signaling in NSCLC and have been proven as promising tumor gene therapeutic targets because of their abilities to promote the proliferation of NSCLC cells. Increasing the lncRNA SNHG1 expression could also enhance cell proliferation[97]. The expression of SNHG1 has a negative correlation with miR-101-3p, and SOX9 could act as the target of miR-101-3p. Given that SOX9 has a great tendency to control oncogenic Wnt/ $\beta$-catenin signaling[98-100], SNHG1 promotes NSCLC tumorigenesis and progression via the miR-101-3p/SOX9/Wnt/ $\beta$-catenin axis. However, lncRNA AK126698, which is usually downregulated, can inhibit the proliferation and migration and increase the apoptosis of NSCLC cells by repressing the Wnt/ $\beta$-catenin signaling pathway through targeting FZD8 in a complex[101]. Furthermore, chemosensitivity is induced through the inactivation of the Wnt/ $\beta$-catenin pathway. LncRNA Meg3 regulates the specificity of p53 transcriptional activation[102], thereby inhibiting $\beta$-catenin/survivin of cancer cells to regulate cisplatin resistance in NSCLC[103]. 
Table 2. Wnt-related IncRNAs in several cancers

\begin{tabular}{|c|c|c|c|c|c|}
\hline LncRNA & Cancer type & $\begin{array}{l}\text { Expression } \\
\text { pattern }\end{array}$ & $\begin{array}{l}\text { Interaction with } \\
\text { WNT signaling }\end{array}$ & Cancer phenotype & Reference \\
\hline AFAP1-AS1 & Tongue squamous cell carcinoma & Up & Activate & $\begin{array}{l}\text { Promote proliferation invasion, migration and } \\
\text { clone formation }\end{array}$ & [112] \\
\hline ARSR & Epithelial ovarian cancer & $\mathrm{Up}$ & Activate & Promote proliferation and invasion & [113] \\
\hline CCAT1 & Cervical cancer & $\mathrm{Up}$ & Activate & Promote proliferation and inhibit apoptosis of cells & [114] \\
\hline CCAT2 & Breast cancer, Epithelial ovarian carcinoma & $\mathrm{Up}$ & Activate & Promote proliferation and invasion & $\begin{array}{l}{[115]} \\
{[116]}\end{array}$ \\
\hline CILA1 & Tongue squamous cell carcinoma & $\mathrm{Up}$ & Activate & Promote EMT, invasion and chemo-resistance & [117] \\
\hline DUXAP8 & Esophageal squamous cell cancer & Up & Activate & Promote proliferation and invasion & [118] \\
\hline HOTAIR & $\begin{array}{l}\text { Esophageal squamous cell carcinoma, } \\
\text { Pancreatic ductal adenocarcinoma, Ovarian } \\
\text { cancer }\end{array}$ & $\mathrm{Up}$ & Activate & Promote migration, invasion and chemoresistance & $\begin{array}{l}{[119]} \\
{[120]} \\
{[121]}\end{array}$ \\
\hline HOXD-AS1 & Epithelial ovarian cancer & $\mathrm{Up}$ & Activate & Promote proliferation, invasion and EMT & [122] \\
\hline Linc-ROR & Ovarian cancer & $\mathrm{Up}$ & Activate & Promote proliferation, metastasis and EMT & [123] \\
\hline SNHG1 & Osteosarcoma & $\mathrm{Up}$ & Activate & Promote proliferation, migration and EMT & [124] \\
\hline SNHG20 & Ovarian cancer & $\mathrm{Up}$ & Activate & Promote proliferation & [125] \\
\hline SOX4 & Osteosarcoma & $\mathrm{Up}$ & Activate & Promote proliferation and migration & [126] \\
\hline UCA1 & $\begin{array}{l}\text { Oral squamous cell carcinoma, } \\
\text { Breast cancer, melanoma }\end{array}$ & $\mathrm{Up}$ & Activate & $\begin{array}{l}\text { Promote proliferation, metastasis and tamoxifen } \\
\text { resistance }\end{array}$ & $\begin{array}{l}{[127]} \\
{[128,129]}\end{array}$ \\
\hline MEG3 & Retinoblastoma, Melanoma & Down & Repress & Inhibit proliferation and promote apoptosis & {$[130][131]$} \\
\hline PTCSC3 & Glioma & Down & Repress & Inhibit proliferation and invasion & [132] \\
\hline UCA1 & $\begin{array}{l}\text { Esophageal squamous-cell } \\
\text { carcinoma }\end{array}$ & Down & Repress & $\begin{array}{l}\text { Inhibit proliferation, migration, invasion, and cell } \\
\text { cycle progression }\end{array}$ & [133] \\
\hline
\end{tabular}

\section{Wnt pathway-related lncRNAs and bladder cancer}

As one of the most common urological malignancy worldwide[104], bladder cancer is the fourth most common cancer in man and the ninth most common cancer in women. Given the high recurrence rate, poor prognosis, and low 5-year overall survival rates of bladder cancer[105], scholars should explore the molecular mechanisms underlying its tumorigenesis to identify novel therapeutic targets and molecular biomarkers for treatment. Liang Ying reported[106] that IncRNA MALAT-1 might contribute to the EMT and metastasis of bladder cancer in vitro by activating the Wnt/ $\beta$-catenin signaling pathway. Increasing the expression of lncRNAs associated with the Wnt/ $\beta$-catenin signaling pathway regulates the progression and chemoresistance of bladder cancer. Overexpression of XIST can function as a sponge of miR-139-5p to regulate the expression of Wnt1, which activates the Wnt/ $\beta$-catenin signaling pathway and functions in the pathogenesis of bladder cancer. Moreover, lncRNA TUG1[107] and UCA1[108] affect the outcomes of chemotherapy; TUG1 knockdown suppresses $\beta$-catenin expression to inhibit Dox resistance and upregulates UCA1 increased Wnt6 expression to induce cisplatin resistance. Hence, the interaction between lncRNA and WNT signaling plays an important role in bladder cancer chemoresistance. In addition to normal physiological functions, lncRNAs are deregulated in bladder cancer development and progression. LncRNA CASC2 is expressed at a low level in bladder cancer and inhibits $W n t / \beta$-catenin signaling by decreasing the expression of $\beta$-catenin, thereby suppressing the growth, migration, and invasion of bladder cancer cells[109].

\section{Conclusion and future perspective}

LncRNAs are a highly heterogeneous group of transcripts that regulate gene expression and various aspects of cellular homeostasis, including tumor cell proliferation, survival, and migration or genomic stability through diverse mechanisms[110]. Our collected data confirm that lncRNAs have great roles in the progression of tumorigenesis and, similar to protein-coding genes, are associated with a variety of cell signaling pathways. Although several pathways exist through which lncRNAs may be targeted to modulate biological cellular processes, the Wnt signaling pathway is a common feature of tumors and plays important roles in tumor progression and metastasis of different cancer types[111]. The transcription of lncRNAs can provide signals of malignant transformation through their interactions with other cellular macromolecules, including DNA, protein, and RNA, to activate or suppress the Wnt $/ \beta$-catenin signaling pathway. Hence, IncRNAs considerably influence the pathology of different cancers. Some Wnt-related lncRNAs that have been associated with cancer but are not reported previously are listed in Table 2. Moreover, the same lncRNAs may have diverse ways to target $\mathrm{Wnt} / \beta$-catenin signaling in the progression of different carcinomas because of their highly tissue- and cell type-specific 
expression patterns. The interactions between Wnt/ $\beta$-catenin signaling and lncRNAs have important roles in the development of tumorigenesis. The results provide evidence that lncRNA-Wnt/ $\beta$-catenin signaling can be a potential target for cancer therapy and a diagnostic indicator to predict responses to treatments.

In summary, Wnt pathway-related lncRNAs are differentially modulated in different types of cancer and the expression of downstream genes is correlated with tumorigenesis, tumor aggressiveness, and stages. The network of lncRNAs and Wnt/ $\beta$-catenin signaling exhibits potential for discovery of novel diagnostics and therapeutics for cancer. Given that lncRNAs-Wnt pathway increases the therapeutic sensitivity of tumors by modulating several critical genes that confer resistance, the underlying mechanism of such network should be further explored to improve therapeutic efficacy and develop combination therapies. Although a large number of lncRNAs are identified to be associated with $\mathrm{Wnt} / \beta$-catenin signaling in various types of cancers by binding to miRNAs or by regulating the key factors of Wnt signaling, such as $\beta$-catenin and TCF, some Wnt pathway-related lncRNAs and their detailed functions and specificity remain unclear and should be investigated in the future.

\section{Acknowledgments}

Funding: This study was funded by grants from the National Natural Science Foundation of China (No. 81472663, 81502280, 81672845 and 81872304), the Education Department of Jiangsu Province (No. 15KJA320006), and the Project of Invigorating Health Care through Science, Technology and Education from Jiangsu Province.

\section{Ethical approval}

This article does not contain any studies with human participants or animals performed by any of the authors.

\section{Conflict of interests}

The authors declare that they have no conflict of interest in this study.

\section{References}

1. Spizzo R, Almeida M I, Colombatti A, et al. Long non-coding RNAs and cancer: a new frontier of translational research? Oncogene. 2012; 31(43): 4577-87.

2. Shi J, Dong B, Cao J, et al. Long non-coding RNA in glioma: signaling pathways. Oncotarget. 2017; 8(16): 27582-92.

3. Dykes I M, Emanueli C. Transcriptional and Post-transcriptional Gene Regulation by Long Non-coding RNA. Genomics Proteomics Bioinformatics. 2017; 15(3): 177-86.

4. Deniz E, Erman B. Long noncoding RNA (lincRNA), a new paradigm in gene expression control. Funct Integr Genomics. 2017; 17(2-3): 135-43.

5. Brannan C I, Dees E C, Ingram R S, et al. The product of the H19 gene may function as an RNA. Mol Cell Biol. 1990; 10(1): 28-36.
6. Kwok Z H, Tay Y. Long noncoding RNAs: lincs between human health and disease. Biochem Soc Trans. 2017; 45(3): 805-12.

7. Li D Y, Busch A, Jin H, et al. H19 Induces Abdominal Aortic Aneurysm Development and Progression. Circulation. 2018;

8. Zhang W, Li Y, Wang P. Long non-coding RNA-ROR aggravates myocardial ischemia/reperfusion injury. Braz J Med Biol Res. 2018; 51(6): e6555.

9. Fan X, Yuan J, Xie J, et al. Long non-protein coding RNA DANCR functions as a competing endogenous RNA to regulate osteoarthritis progression via miR-577/SphK2 axis. Biochem Biophys Res Commun. 2018; 500(3): 658-64.

10. Chen D, Liu L, Wang K, et al. The role of MALAT-1 in the invasion and metastasis of gastric cancer. Scand J Gastroenterol. 2017; 52(6-7): 790-6.

11. Svoboda M, Slyskova J, Schneiderova M, et al. HOTAIR long non-coding RNA is a negative prognostic factor not only in primary tumors, but also in the blood of colorectal cancer patients. Carcinogenesis. 2014; 35(7): 1510-5.

12. Ma Y, Yang $Y$, Wang F, et al. Long non-coding RNA CCAL regulates colorectal cancer progression by activating Wnt/beta-catenin signalling pathway via suppression of activator protein 2alpha. Gut. 2016; 65(9): 1494-504.

13. Gupta R A, Shah N, Wang K C, et al. Long non-coding RNA HOTAIR reprograms chromatin state to promote cancer metastasis. Nature. 2010; 464(7291): 1071-6.

14. Bhan A, Soleimani M, Mandal S S. Long Noncoding RNA and Cancer: A New Paradigm. Cancer Res. 2017; 77(15): 3965-81.

15. Wang K C, Chang H Y. Molecular mechanisms of long noncoding RNAs. Mol Cell. 2011; 43(6): 904-14.

16. Sun $\mathrm{L}$, Jiang $\mathrm{C}, \mathrm{Xu} \mathrm{C}$, et al. Down-regulation of long non-coding RNA RP11-708H21.4 is associated with poor prognosis for colorectal cancer and promotes tumorigenesis through regulating AKT/mTOR pathway. Oncotarget. 2017; 8(17): 27929-42.

17. Yu T, Zhao Y, Hu Z, et al. MetaLnc9 Facilitates Lung Cancer Metastasis via a PGK1-Activated AKT/mTOR Pathway. Cancer Res. 2017; 77(21): 5782-94.

18. Crea F, Clermont P L, Parolia A, et al. The non-coding transcriptome as a dynamic regulator of cancer metastasis. Cancer Metastasis Rev. 2014; 33(1): $1-16$.

19. Nusse R, Varmus H E. Many tumors induced by the mouse mammary tumor virus contain a provirus integrated in the same region of the host genome. Cell. 1982; 31(1): 99-109.

20. Peng $Y$, Zhang $X$, Feng $X$, et al. The crosstalk between microRNAs and the Wnt/beta-catenin signaling pathway in cancer. Oncotarget. 2017; 8(8): 14089-106.

21. Nusse R, Clevers H. Wnt/beta-Catenin Signaling, Disease, and Emerging Therapeutic Modalities. Cell. 2017; 169(6): 985-99.

22. Liu C, Kato Y, Zhang Z, et al. beta-Trcp couples beta-catenin phosphorylation-degradation and regulates Xenopus axis formation. Proc Natl Acad Sci U S A. 1999; 96(11): 6273-8.

23. Valenta T, Hausmann G, Basler K. The many faces and functions of beta-catenin. EMBO J. 2012; 31(12): 2714-36.

24. Rao T P, Kuhl M. An updated overview on Wnt signaling pathways: a prelude for more. Circ Res. 2010; 106(12): 1798-806.

25. Anastas J N, Moon R T. WNT signalling pathways as therapeutic targets in cancer. Nat Rev Cancer. 2013; 13(1): 11-26.

26. Derksen $\mathrm{P}$ W, Tjin E, Meijer H P, et al. Illegitimate WNT signaling promotes proliferation of multiple myeloma cells. Proc Natl Acad Sci U S A. 2004; 101(16): 6122-7.

27. Yeung J, Esposito M T, Gandillet A, et al. beta-Catenin mediates the establishment and drug resistance of MLL leukemic stem cells. Cancer Cell. 2010; 18(6): 606-18

28. Yook J I, Li X Y, Ota I, et al. A Wnt-Axin2-GSK3beta cascade regulates Snail1 activity in breast cancer cells. Nat Cell Biol. 2006; 8(12): 1398-406.

29. Xie $X$, Tang B, Xiao Y F, et al. Long non-coding RNAs in colorectal cancer. Oncotarget. 2016; 7(5): 5226-39.

30. Xiao Z, Qu Z, Chen Z, et al. LncRNA HOTAIR is a Prognostic Biomarker for the Proliferation and Chemoresistance of Colorectal Cancer via MiR-203a-3p-Mediated Wnt/ss-Catenin Signaling Pathway. Cell Physiol Biochem. 2018; 46(3): 1275-85.

31. Lu Y, Zhao X, Liu $Q$, et al. IncRNA MIR100HG-derived miR-100 and miR-125b mediate cetuximab resistance via Wnt/beta-catenin signaling. Nat Med. 2017; 23(11): 1331-41.

32. Bafico A, Liu G, Yaniv A, et al. Novel mechanism of Wnt signalling inhibition mediated by Dickkopf-1 interaction with LRP6/Arrow. Nat Cell Biol. 2001; 3(7): 683-6.

33. Nakamura R E, Hackam A S. Analysis of Dickkopf3 interactions with Wnt signaling receptors. Growth Factors. 2010; 28(4): 232-42.

34. de Lau W, Peng W C, Gros P, et al. The R-spondin/Lgr5/Rnf43 module: regulator of Wnt signal strength. Genes Dev. 2014; 28(4): 305-16.

35. Jiang $\mathrm{X}$, Charlat $\mathrm{O}$, Zamponi $\mathrm{R}$, et al. Dishevelled promotes Wnt receptor degradation through recruitment of ZNRF3/RNF43 E3 ubiquitin ligases. Mol Cell. 2015; 58(3): 522-33.

36. Cruciat $\mathrm{C} M$, Niehrs C. Secreted and transmembrane wnt inhibitors and activators. Cold Spring Harb Perspect Biol. 2013; 5(3): a015081.

37. MacDonald B T, Tamai K, He X. Wnt/beta-catenin signaling: components, mechanisms, and diseases. Dev Cell. 2009; 17(1): 9-26.

38. Sun N, Zhang G, Liu Y. Long non-coding RNA XIST sponges miR-34a to promotes colon cancer progression via Wnt/beta-catenin signaling pathway. Gene. 2018; 
39. Zhang Z, Zhou C, Chang $\mathrm{Y}$, et al. Long non-coding RNA CASC11 interacts with hnRNP-K and activates the WNT/beta-catenin pathway to promote growth and metastasis in colorectal cancer. Cancer Lett. 2016; 376(1): 62-73.

40. $\mathrm{Li} \mathrm{T}$, Zhu J, Wang $\mathrm{X}$, et al. Long non-coding RNA IncTCF7 activates the Wnt/beta-catenin pathway to promote metastasis and invasion in colorectal cancer. Oncol Lett. 2017; 14(6): 7384-90.

41. Lin J, Tan X, Qiu L, et al. Long Noncoding RNA BC032913 as a Novel Therapeutic Target for Colorectal Cancer that Suppresses Metastasis by Upregulating TIMP3. Mol Ther Nucleic Acids. 2017; 8:469-81.

42. Wang G, Li Z, Zhao Q, et al. LincRNA-p21 enhances the sensitivity of radiotherapy for human colorectal cancer by targeting the Wnt/beta-catenin signaling pathway. Oncol Rep. 2014; 31(4): 1839-45.

43. Shan Z, An N, Qin J, et al. Long non-coding RNA Linc00675 suppresses cell proliferation and metastasis in colorectal cancer via acting on miR-942 and Wnt/beta-catenin signaling. Biomed Pharmacother. 2018; 101:769-76.

44. Ouyang S, Zheng X, Zhou X, et al. LncRNA BCAR4 promotes colon cancer progression via activating Wnt/beta-catenin signaling. Oncotarget. 2017; 8(54): 92815-26.

45. Liu X B, Han C, Sun C Z. Long non-coding RNA DLEU7-AS1 promotes the occurrence and development of colorectal cancer via Wnt/beta-catenin pathway. Eur Rev Med Pharmacol Sci. 2018; 22(1): 110-7.

46. Zhang $X$, Xiong $Y$, Tang $F$, et al. Long noncoding RNA HNF1A-AS1 indicates a poor prognosis of colorectal cancer and promotes carcinogenesis via activation of the Wnt/beta-catenin signaling pathway. Biomed Pharmacother. 2017; 96:877-83.

47. Wu K F, Liang W C, Feng L, et al. H19 mediates methotrexate resistance in colorectal cancer through activating Wnt/beta-catenin pathway. Exp Cell Res. 2017; 350(2): 312-7.

48. Qi H, Wang J, Wang F, et al. Long non-coding RNA SNHG1 promotes cell proliferation and tumorigenesis in colorectal cancer via Wnt/beta-catenin signaling. Pharmazie. 2017; 72(7): 395-401.

49. Yuan Z, Yu X, Ni B, et al. Overexpression of long non-coding RNA-CTD903 inhibits colorectal cancer invasion and migration by repressing Wnt/beta-catenin signaling and predicts favorable prognosis. Int J Oncol. 2016; 48(6): 2675-85

50. Clevers H. Wnt/ beta-catenin signaling in development and disease. Cell. 2006; 127(3): 469-80.

51. Hoffmeyer K, Raggioli A, Rudloff $\mathrm{S}$, et al. Wnt/beta-catenin signaling regulates telomerase in stem cells and cancer cells. Science. 2012; 336(6088): 1549-54.

52. Li V S, Ng S S, Boersema P J, et al. Wnt signaling through inhibition of beta-catenin degradation in an intact Axin1 complex. Cell. 2012; 149(6): 1245-56.

53. Deschene E R, Myung $\mathrm{P}$, Rompolas $\mathrm{P}$, et al. beta-Catenin activation regulates tissue growth non-cell autonomously in the hair stem cell niche. Science. 2014; 343(6177): 1353-6.

54. Fu X, Lin J, Qin F, et al. LncAPC drives Wnt/ beta-catenin activation and liver TIC self-renewal through EZH2 mediated APC transcriptional inhibition. Mol Carcinog. 2018; 57(3): 408-18

55. Xu D, Yang F, Yuan J H, et al. Long noncoding RNAs associated with liver regeneration 1 accelerates hepatocyte proliferation during liver regeneration by activating Wnt/beta-catenin signaling. Hepatology. 2013; 58(2): 739-51.

56. Wang $\mathrm{Y}, \mathrm{He} \mathrm{L}, \mathrm{Du} \mathrm{Y}$, et al. The long noncoding RNA IncTCF7 promotes self-renewal of human liver cancer stem cells through activation of Wnt signaling. Cell Stem Cell. 2015; 16(4): 413-25.

57. Helming K C, Wang X, Roberts C W M. Vulnerabilities of mutant SWI/SNF complexes in cancer. Cancer Cell. 2014; 26(3): 309-17.

58. Wilson B G, Roberts C W. SWI/SNF nucleosome remodellers and cancer. Nat Rev Cancer. 2011;11(7): 481-92.

59. Fu X, Zhu X, Qin F, et al. Linc00210 drives Wnt/beta-catenin signaling activation and liver tumor progression through CTNNBIP1-dependent manner. Mol Cancer. 2018; 17(1): 73 .

60. Zhu P, Wang Y, Huang G, et al. Inc-beta-Catm elicits EZH2-dependent beta-catenin stabilization and sustains liver CSC self-renewal. Nat Struct Mol Biol. 2016; 23(7): 631-9.

61. McTyre E, Lucas J T, Helis C, et al. Outcomes for Anaplastic Glioma Treated With Radiation Therapy With or Without Concurrent Temozolomide. Am J Clin Oncol. 2017;

62. Zhang $\mathrm{Y}, \mathrm{Yu} \mathrm{X}, \mathrm{Chen} \mathrm{L}$, et al. EZH2 overexpression is associated with poor prognosis in patients with glioma. Oncotarget. 2017; 8(1): 565-73.

63. Li Y, Zhu G, Zeng W, et al. Long noncoding RNA AB073614 promotes the malignance of glioma by activating Wnt/beta-catenin signaling through downregulating SOX7. Oncotarget. 2017; 8(39): 65577-87.

64. Guo H, Hu G, Yang Q, et al. Knockdown of long non-coding RNA CCAT2 suppressed proliferation and migration of glioma cells. Oncotarget. 2016; 7(49): 81806-14.

65. Zhang H, Wei D L, Wan L, et al. Highly expressed lncRNA CCND2-AS1 promotes glioma cell proliferation through Wnt/beta-catenin signaling. Biochem Biophys Res Commun. 2017; 482(4): 1219-25.

66. Li J, Zhou L. Overexpression of IncRNA DANCR positively affects progression of glioma via activating Wnt/beta-catenin signaling. Biomed Pharmacother. 2018; 102:602-7.

67. Ong M S, Cai W, Yuan Y, et al. 'Lnc'-ing Wnt in female reproductive cancers: therapeutic potential of long non-coding RNAs in Wnt signalling. Br J Pharmacol. 2017; 174(24): 4684-700.
68. Choudhry $\mathrm{H}$, Albukhari $\mathrm{A}$, Morotti $\mathrm{M}$, et al. Tumor hypoxia induces nuclear paraspeckle formation through HIF-2alpha dependent transcriptional activation of NEAT1 leading to cancer cell survival. Oncogene. 2015; 34(34): 4546.

69. Wu Y, Yang L, Zhao J, et al. Nuclear-enriched abundant transcript 1 as a diagnostic and prognostic biomarker in colorectal cancer. Mol Cancer. 2015; 14:191.

70. Chakravarty D, Sboner A, Nair S S, et al. The oestrogen receptor alpha-regulated lncRNA NEAT1 is a critical modulator of prostate cancer. Nat Commun. 2014; 5:5383.

71. Chen Q, Cai J, Wang Q, et al. Long Noncoding RNA NEAT1, Regulated by the EGFR Pathway, Contributes to Glioblastoma Progression Through the WNT/beta-Catenin Pathway by Scaffolding EZH2. Clin Cancer Res. 2018; 24(3): 684-95.

72. Jho E H, Zhang T, Domon C, et al. Wnt/beta-catenin/Tcf signaling induces the transcription of Axin2, a negative regulator of the signaling pathway. Mol Cell Biol. 2002; 22(4): 1172-83.

73. Tago K, Nakamura T, Nishita M, et al. Inhibition of Wnt signaling by ICAT, a novel beta-catenin-interacting protein. Genes Dev. 2000; 14(14): 1741-9.

74. Domingues $\mathrm{M}$ J, Rambow F, Job $\mathrm{B}$, et al. beta-catenin inhibitor ICAT modulates the invasive motility of melanoma cells. Cancer Res. 2014; 74(7): 1983-95.

75. Siegel R L, Miller K D, Fedewa S A, et al. Colorectal cancer statistics, 2017. CA Cancer J Clin. 2017; 67(3): 177-93.

76. Karimi P, Islami F, Anandasabapathy S, et al. Gastric cancer: descriptive epidemiology, risk factors, screening, and prevention. Cancer Epidemiol Biomarkers Prev. 2014; 23(5): 700-13.

77. Cheng C, Qin Y, Zhi Q, et al. Knockdown of long non-coding RNA HOTAIR inhibits cisplatin resistance of gastric cancer cells through inhibiting the PI3K/Akt and Wnt/beta-catenin signaling pathways by up-regulating miR-34a. Int J Biol Macromol. 2018; 107(Pt B): 2620-9.

78. Wu X, Zhang P, Zhu $\mathrm{H}$, et al. Long noncoding RNA FEZF1-AS1 indicates a poor prognosis of gastric cancer and promotes tumorigenesis via activation of Wnt signaling pathway. Biomed Pharmacother. 2017; 96:1103-8.

79. Luo $Y$, Tan $W$, Jia W, et al. The long non-coding RNA LINC01606 contributes to the metastasis and invasion of human gastric cancer and is associated with Wnt/beta-catenin signaling. Int J Biochem Cell Biol. 2018; 103:125-34.

80. Yang X Z, Cheng T T, He Q J, et al. LINC01133 as ceRNA inhibits gastric cancer progression by sponging miR-106a-3p to regulate APC expression and the Wnt/beta-catenin pathway. Mol Cancer. 2018; 17(1): 126.

81. Zhao Y X, Liu J F, Sun W J, et al. Long non-coding RNA-ENST00000434223 suppresses tumor progression in gastric cancer cells through the Wnt/beta-catenin signaling pathway. Int J Biol Macromol. 2018; 120(Pt A): 491-501.

82. Askarian-Amiri M E, Crawford J, French J D, et al. SNORD-host RNA Zfas1 is a regulator of mammary development and a potential marker for breast cancer. RNA. 2011; 17(5): 878-91.

83. Li T, Xie J, Shen C, et al. Amplification of Long Noncoding RNA ZFAS1 Promotes Metastasis in Hepatocellular Carcinoma. Cancer Res. 2015; 75(15): 3181-91.

84. Thorenoor N, Faltejskova-Vychytilova P, Hombach S, et al. Long non-coding RNA ZFAS1 interacts with CDK1 and is involved in p53-dependent cell cycle control and apoptosis in colorectal cancer. Oncotarget. 2016; 7(1): 622-37.

85. Pan L, Liang W, Fu M, et al. Exosomes-mediated transfer of long noncoding RNA ZFAS1 promotes gastric cancer progression. J Cancer Res Clin Oncol. 2017; 143(6): 991-1004.

86. Nie F, Yu X, Huang M, et al. Long noncoding RNA ZFAS1 promotes gastric cancer cells proliferation by epigenetically repressing KLF2 and NKD2 expression. Oncotarget. 2017; 8(24):38227-38.

87. Xu W, He L, Li Y, et al. Silencing of lncRNA ZFAS1 inhibits malignancies by blocking Wnt/beta-catenin signaling in gastric cancer cells. Biosci Biotechnol Biochem. 2018; 82(3): 456-65.

88. $\mathrm{Lu} \mathrm{Y}, \mathrm{Yu} \mathrm{Y}, \mathrm{Zhu} \mathrm{Z}$, et al. Identification of a new target region by loss of heterozygosity at $5 \mathrm{p} 15.33$ in sporadic gastric carcinomas: genotype and phenotype related. Cancer Lett. 2005; 224(2): 329-37.

89. Xu S F, Li D P, Peng Z H. [Refined mapping of heterozygosity loss on chromosome 5 p15 in sporadic colorectal cancer]. Zhonghua Zhong Liu Za Zhi. 2008; 30(6): 413-7.

90. Zeng $\mathrm{X}$, Huang H, Tamai K, et al. Initiation of Wnt signaling: control of Wnt coreceptor Lrp6 phosphorylation/activation via frizzled, dishevelled and axin functions. Development. 2008; 135(2): 367-75.

91. Siegel R, Ward E, Brawley O, et al. Cancer statistics, 2011: the impact of eliminating socioeconomic and racial disparities on premature cancer deaths. CA Cancer J Clin. 2011; 61(4): 212-36.

92. Siegel R L, Miller K D, Jemal A. Cancer statistics, 2018. CA Cancer J Clin. 2018; 68(1): 7-30.

93. Raungrut $\mathrm{P}$, Wongkotsila A, Lirdprapamongkol $\mathrm{K}$, et al. Prognostic significance of 14-3-3gamma overexpression in advanced non-small cell lung cancer. Asian Pac J Cancer Prev. 2014; 15(8): 3513-8.

94. Wang Y, Zhou J, Xu Y J, et al. Long non-coding RNA LINC00968 acts as oncogene in NSCLC by activating the Wnt signaling pathway. J Cell Physiol. 2018; 233(4): 3397-406.

95. Sun S J, Lin $\mathrm{Q}, \mathrm{Ma} J \mathrm{X}$, et al. Long non-coding RNA NEAT1 acts as oncogene in NSCLC by regulating the Wnt signaling pathway. Eur Rev Med Pharmacol Sci. 2017; 21(3): 504-10. 
96. Rong L, Zhao R, Lu J. Highly expressed long non-coding RNA FOXD2-AS1 promotes non-small cell lung cancer progression via Wnt/beta-catenin signaling. Biochem Biophys Res Commun. 2017; 484(3): 586-91.

97. Cui $\mathrm{Y}$, Zhang F, Zhu C, et al. Upregulated IncRNA SNHG1 contributes to progression of non-small cell lung cancer through inhibition of miR-101-3p and activation of Wnt/beta-catenin signaling pathway. Oncotarget. 2017; 8(11): 17785-94.

98. Santos J C, Carrasco-Garcia E, Garcia-Puga M, et al. SOX9 Elevation Acts with Canonical WNT Signaling to Drive Gastric Cancer Progression. Cancer Res. 2016; 76(22): 6735-46

99. $\mathrm{Ma} \mathrm{F}, \mathrm{Ye} \mathrm{H}, \mathrm{He} \mathrm{H} \mathrm{H}$, et al. SOX9 drives WNT pathway activation in prostate cancer. J Clin Invest. 2016; 126(5): 1745-58.

100. Prevostel C, Rammah-Bouazza C, Trauchessec H, et al. SOX9 is an atypical intestinal tumor suppressor controlling the oncogenic Wnt/ss-catenin signaling. Oncotarget. 2016; 7(50): 82228-43.

101. Fu X, Li H, Liu C, et al. Long noncoding RNA AK126698 inhibits proliferation and migration of non-small cell lung cancer cells by targeting Frizzled- 8 and suppressing Wnt/beta-catenin signaling pathway. Onco Targets Ther. 2016; 9:3815-27.

102. Zhou Y, Zhong Y, Wang Y, et al. Activation of p53 by MEG3 non-coding RNA. J Biol Chem. 2007; 282(34): 24731-42.

103. Xia Y, He Z, Liu B, et al. Downregulation of Meg3 enhances cisplatin resistance of lung cancer cells through activation of the WNT/beta-catenin signaling pathway. Mol Med Rep. 2015; 12(3): 4530-7.

104. Torre L A, Bray F, Siegel R L, et al. Global cancer statistics, 2012. CA Cancer J Clin. 2015; 65(2): 87-108.

105. Chou R, Selph S S, Buckley D I, et al. Treatment of muscle-invasive bladder cancer: A systematic review. Cancer. 2016; 122(6): 842-51.

106. Ying L, Chen $Q$, Wang Y, et al. Upregulated MALAT-1 contributes to bladder cancer cell migration by inducing epithelial-to-mesenchymal transition. Mol Biosyst. 2012; 8(9): 2289-94.

107. Xie D, Zhang H, Hu X, et al. Knockdown of long non-coding RNA Taurine Up-Regulated 1 inhibited doxorubicin resistance of bladder urothelial carcinoma via Wnt/beta-catenin pathway. Oncotarget. 2017; 8(51): 88689-96.

108. Fan $\mathrm{Y}$, Shen B, Tan $\mathrm{M}$, et al. Long non-coding RNA UCA1 increases chemoresistance of bladder cancer cells by regulating Wnt signaling. FEBS J. 2014; 281(7): 1750-8.

109. Pei Z, Du X, Song Y, et al. Down-regulation of lncRNA CASC2 promotes cell proliferation and metastasis of bladder cancer by activation of the Wnt/beta-catenin signaling pathway. Oncotarget. 2017; 8(11): 18145-53.

110. Huarte M. The emerging role of lncRNAs in cancer. Nat Med. 2015; 21(11): 1253-61.

111. Shiah S G, Shieh Y S, Chang J Y. The Role of Wnt Signaling in Squamous Cell Carcinoma. J Dent Res. 2016; 95(2): 129-34.

112. Wang Z Y, Hu M, Dai M H, et al. Upregulation of the long non-coding RNA AFAP1-AS1 affects the proliferation, invasion and survival of tongue squamous cell carcinoma via the Wnt/beta-catenin signaling pathway. Mol Cancer. 2018; 17(1): 3.

113. Shu C, Yan D, Mo Y, et al. Long noncoding RNA lncARSR promotes epithelial ovarian cancer cell proliferation and invasion by association with HuR and miR-200 family. Am J Cancer Res. 2018; 8(6): 981-92.

114. Zhang J, Gao Y. CCAT-1 promotes proliferation and inhibits apoptosis of cervical cancer cells via the Wnt signaling pathway. Oncotarget. 2017; 8(40): 68059-70.

115. Cai Y, He J, Zhang D. Long noncoding RNA CCAT2 promotes breast tumor growth by regulating the Wnt signaling pathway. Onco Targets Ther. 2015; 8:2657-64

116. Wang B, Liu M, Zhuang R, et al. Long non-coding RNA CCAT2 promotes epithelial-mesenchymal transition involving Wnt/beta-catenin pathway in epithelial ovarian carcinoma cells. Oncol Lett. 2018; 15(3): 3369-75.

117. Lin Z, Sun L, Xie S, et al. Chemotherapy-Induced Long Non-coding RNA 1 Promotes Metastasis and Chemo-Resistance of TSCC via the Wnt/beta-Catenin Signaling Pathway. Mol Ther. 2018;

118. Xu L J, Yu X J, Wei B, et al. Long non-coding RNA DUXAP8 regulates proliferation and invasion of esophageal squamous cell cancer. Eur Rev Med Pharmacol Sci. 2018; 22(9): 2646-52.

119. Ge X S, Ma H J, Zheng X H, et al. HOTAIR, a prognostic factor in esophageal squamous cell carcinoma, inhibits WIF-1 expression and activates Wnt pathway. Cancer Sci. 2013; 104(12): 1675-82.

120. Jiang Y, Li Z, Zheng S, et al. The long non-coding RNA HOTAIR affects the radiosensitivity of pancreatic ductal adenocarcinoma by regulating the expression of Wnt inhibitory factor 1. Tumour Biol. 2016; 37(3): 3957-67.

121. Li J, Yang S, Su N, et al. Overexpression of long non-coding RNA HOTAIR leads to chemoresistance by activating the Wnt/beta-catenin pathway in human ovarian cancer. Tumour Biol. 2016; 37(2): 2057-65.

122. Zhang Y, Dun Y, Zhou S, et al. LncRNA HOXD-AS1 promotes epithelial ovarian cancer cells proliferation and invasion by targeting miR-133a-3p and activating Wnt/beta-catenin signaling pathway. Biomed Pharmacother. 2017; 96:1216-21.

123. Lou Y, Jiang H, Cui Z, et al. Linc-ROR induces epithelial-to-mesenchymal transition in ovarian cancer by increasing Wnt/beta-catenin signaling. Oncotarget. 2017; 8(41): 69983-94

124. Jiang Z, Jiang C, Fang J. Up-regulated lnc-SNHG1 contributes to osteosarcoma progression through sequestration of miR-577 and activation of
WNT2B/Wnt/beta-catenin pathway. Biochem Biophys Res Commun. 2018; 495(1): 238-45.

125. He S, Zhao Y, Wang X, et al. Up-regulation of long non-coding RNA SNHG20 promotes ovarian cancer progression via Wnt/beta-catenin signaling. Biosci Rep. 2018; 38(1): BSR20170681.

126. Tian Z, Yang G, Jiang P, et al. Long non-coding RNA Sox4 promotes proliferation and migration by activating Wnt/beta-catenin signaling pathway in osteosarcoma. Pharmazie. 2017; 72(9): 537-42.

127. Yang Y T, Wang Y F, Lai J Y, et al. Long non-coding RNA UCA1 contributes to the progression of oral squamous cell carcinoma by regulating the WNT/beta-catenin signaling pathway. Cancer Sci. 2016; 107(11): 1581-9.

128. Liu H, Wang G, Yang L, et al. Knockdown of Long Non-Coding RNA UCA1 Increases the Tamoxifen Sensitivity of Breast Cancer Cells through Inhibition of Wnt/beta-Catenin Pathway. PLoS One. 2016; 11(12): e0168406.

129. Xiao C, Wu C H, Hu H Z. LncRNA UCA1 promotes epithelial-mesenchymal transition (EMT) of breast cancer cells via enhancing Wnt/beta-catenin signaling pathway. Eur Rev Med Pharmacol Sci. 2016; 20(13): 2819-24.

130. Gao Y, Lu X. Decreased expression of MEG3 contributes to retinoblastoma progression and affects retinoblastoma cell growth by regulating the activity of Wnt/beta-catenin pathway. Tumour Biol. 2016; 37(2): 1461-9.

131. Li P, Gao Y, Li J, et al. LncRNA MEG3 repressed malignant melanoma progression via inactivating Wnt signaling pathway. J Cell Biochem. 2018;

132. Xia S, Ji R, Zhan W. Long noncoding RNA papillary thyroid carcinoma susceptibility candidate 3 (PTCSC3) inhibits proliferation and invasion of glioma cells by suppressing the Wnt/beta-catenin signaling pathway. BMC Neurol. 2017; 17(1): 30

133. Wang X, Gao Z, Liao J, et al. IncRNA UCA1 inhibits esophageal squamous-cell carcinoma growth by regulating the Wnt signaling pathway. J Toxicol Environ Health A. 2016; 79(9-10): 407-18. 